\title{
Research and Application of Micro-arc Oxidation Technology on Tractor Pistons
}

\author{
Dan Yang \\ Jilin Agricultural University, Changchun, 130118, China \\ Email: 1228256215@qq.com
}

Keywords: Micro-arc Oxidation; Characteristic; Influencing Factors; Tractor; Application

\begin{abstract}
The coating obtained by the micro-arc oxidation technology has a strong ability to bind with the substrate, which can improve the properties of materials such as wear and corrosion resistance, thermal shock resistance and insulation. This paper outlined the characteristics of microarc oxidation technology and influencing factors, studied the application of the micro-arc oxidation technology on tractors pistons.
\end{abstract}

\section{Introduction}

Micro-arc oxidation is one of the surface treatment technology that recently arisen at home, it's main used to surface modification on aluminous, magnesium, titanic and their alloy surface, because of the simplexes' of its technical process and the predominant performance of treated surface, the applying prospect is very bullish by inside and outside insider. The conception of MAO came into being in 1950s. But not until 1970s the technology bean to be studied. And it became the focus of research on abroad in 1980s. At present practical research progressed rapidly at abroad, and it tried to be applied in some fields. At this research direction, American, Germany, Japan, Russia are leading the way in the world. Not until 1990s our country bean to research this technology, and compared to the foreign level, our technology far fall behind them. The research on micro-arc. Micro-arc oxidation technology is also known as micro-plasma oxidation, spark discharge anodizing or anodic spark deposition. It is a high-tech set up based on the common anode oxidation technology, currently the international research on it has become increasingly active. But it is different from the ordinary anodic oxidation, as it places $\mathrm{Al}, \mathrm{Ti}, \mathrm{Ta}, \mathrm{Mg}$ and other metal in the bath of a certain electrolyte, after the electrification the surface would immediately produce a very thin insulating oxide film. When the inter-electrode voltage exceeds a critical value, some of the weak parts of the oxide film breakdown, generating the micro-arc discharge phenomenon. During the discharge process, in the surface of the anode sample it existed about 106 / cm 2 sparks.

This happened in a non-Faraday zone. The discharge instantaneous temperature can reach up to $8000 \mathrm{~K}$ or more, producing a ceramic membrane which has a similar performance with the cemented carbide. It had obtained the application in the medical, electronic engineering, vacuum engineering and other fields[3].

\section{The Characteristics of Micro-arc Oxidation Technology}

Micro-arc oxidation technology can form an oxide film with a special function in aluminum, magnesium, titanium and other metal surfaces to improve the corrosion resistance, surface hardness, oxidation resistance of materials and improve the abrasion resistance and other effects. It has the following characteristics.

In situ growth characteristics. The growth process occurred in the discharge point of the microregion, in the beginning stage it is mainly the in situ structure transformation and thickening growth of the oxide film formed on the natural state in a low temperature and the high temperature oxide scale formed during the forming process. It was found that about $70 \%$ of the aluminum oxide layer is present on the surface of the substrate. Thus, the size of the surface of the sample was little changed. 
Uniform growth characteristics. With the insulating properties of aluminum oxide and magnesium oxide, at the same electrical parameters, the thin area is always a priority of breakdown and then grown thicker to ultimately achieve a uniform thickening of the entire sample.

Between the oxide layer and the substrate it exists a relatively thick transition zone. The microarc oxidation ceramic layer has a significant three-layer hierarchical structure, namely loose surface layer, an intermediate density layer and transition layer.

By varying process conditions and adding colloidal particles in the electrolyte, it can easily adjust the characteristics of film microstructure and get new microstructure, in order to achieve functional design film.

Micro-arc oxidation process is simple. It does not require a vacuum or low temperature and less pre-treatment process are required; it's also pollution-free, environmentally friendly and no emission-limiting elements added restrictions. There is no need to precisely control the temperature of the solution, in the solution below $45^{\circ} \mathrm{C}$ it could get good quality ceramic layer.

Wide adaptability of the material. In addition to aluminum, it is also able to produce the ceramic layer on the surface of $\mathrm{Zr}, \mathrm{Ti}, \mathrm{Mg}$, Ta, $\mathrm{Nb}$ and other metals, in particular alloys which is difficult to be handled with conventional anodizing, such as the aluminum alloy with relatively high copper content and cast aluminum and magnesium alloys with high silicon content.

\section{Micro-arc Oxidation Technology Factors}

\section{Electrolyte.}

For the purposes of the same metals or alloys, different electrolyte results in films with different capacity. Properties of the ceramic layer is mainly determined by the component of the electrolyte. Increment in electrolyte concentration will increase the degree of micro-arc oxidation, while adjusting the proportion of additives the film thickness can be increased to varying degrees to optimize surface properties of the film, achieving the purpose of modification of film.But the over large electrolyte concentration would have a corrosive effect on the film. Laboratories usually use weakly alkaline electrolyte, because there are certain hazards to the environment of the acidic electrolyte. For some additives, if the concentration is too small or too big, it would not be conducive to the reaction, such as sodium tungstate; if the concentration is too small, the reaction would be difficult to proceed while if the concentration is too large, the film surface can be passivated thus is not conducive to obtain excellent surface properties. Thus, the component of the electrolyte has a great influence on the preparation and properties of the ceramic layer, and it should select the best suitable additives and electrolyte concentration ratio.

\section{Raw ingredients.}

Due to the different elements it contains, different raw materials will be showing a different film-forming properties. During the micro-arc oxidation process, thickening of the ceramic layer must undergo thermal diffusion particles and colloidal deposition process. And when different metal elements form metal oxide films with different properties, it will be subject to these oxide crystal properties and charging performance. And the current and plasma generation and transmission have a relatively huge impact on the nature of the oxide film. The alloy composition of the Aluminum alloy substrate and the degree of density of the substrate also have a very big impact on the performance of the ceramic membrane produced on it surface. If the silicon content in the Aluminum alloy is high, then the oxide ceramic membrane produced would be relatively thick; if otherwise then it would be thin.

\section{Discharge parameters.}

In the micro-arc oxidation process, the impact of the discharge waveform and its associated parameters of the power on the test is relatively large. In general, the higher the voltage, the greater the film thickness of micro arc oxidation, but the smoothness of the film and dense would decrease; if low voltage is applied, the oxide film may be difficult to generate, or the film generated can not be applied in the industry, thus the selection of the appropriate voltage is very important to the film's performance and it is also the biggest factors that affect energy consumption.With the increase of pulse frequency, the film thickness decreases rapidly, which is almost a linear decline as 
the hardness of the dense layer rapidly declined. But with the increase of pulse rate, the percentage of dense layer is gradually increased.

\section{Processconditions.}

Before micro-arc oxidation test, degreasing treatment should be carried out on the sample, then after washing with deionized water, so it can get rid of the dirt surface of the film to improve the smoothness and compactness of the oxide film layer. And during the test it must have a good cooling system to ensure the smooth progress of micro-arc oxidation.

\section{The Application on a Tractor Pistons}

Aluminum and magnesium alloy are widely used in aviation, aerospace and other fields for its properties such as light weight, high strength, easy molding, etc., but the weaknesses Easy corrosion and low surface hardness etc. have affected its prospects for application. Therefore, the surface protection treatment must be applied in order to reach the requirements. As the traditional aluminum and magnesium alloy material protection methods can not meet the requirements of the parts, the advantages of micro-arc oxidation protection can solve such problems. film

The state of the surface of the parts should be kept the same, to ensure the performance of the

The materials gone through different heat treatment processes such as material forging, casting or heat treatment have very significant different textures. The process parameters can have a great impact on the performance of the films of different materials in different structures, thus different process parameters should be applied to carry out the micro-arc oxidation; at the same time, the partial grinding, polishing and other repair processes of the parts would cause different surface state and affect the performance of films, the surface state of the parts should be kept the same in the processing process to ensure the performance of the film.

\section{Reduce the sharp structure in parts to avoid discharging effect}

In the micro-arc oxidation process, the sharp corners in the parts will produce point discharge effect, causing the local film thicker, coarser and the brittle larger, resulting in poor adhesion of the film, and in subsequent processing and assembly process because of bump and other reasons it can easily have a "block out" phenomenon. Thus the structural design of the parts should be optimized and sharp corners should be minimized to avoid point discharge effect, in order to meet its protective properties.

\section{Select the appropriate film thickness to keep the adhesion and brittleness}

The over-thickness of the parts surface film will result in the decrease in binding force and the increase in brittleness; while when it's too thin the resistance to corrosion and wear protection performance will drop and result in the failure to reach the requirements. In practice, we should select the medium and lower limit of the film thickness in the range allowed in the process in the micro-arc oxidation, which can avoid the decrease in binding force and the increase in brittleness and other issues caused by the over-thickness of the film.

\section{Set the appropriate process parameters to ensure the reliability of quality parts}

Since the micro-arc oxidation stability requirements of process parameters and the electrolyte is very strict, if the electrolyte stability is poor, the film will appear dark spots, white spots, projections and other defects. Therefore further test assessment program should be developed to determine the impact of these defects on the protective properties of the parts and ensure the overall protection performance to meet the requirements and protect the quality and reliability of the parts.

\section{Conclusion}

Micro-arc oxidation technology combines both advantages of the anodic oxide film coating and ceramic coating. As an advanced surface protection methods, it greatly improves the performance of tractor pistons and allows them to be used in more demanding environments. Currently, microarc oxidation technology is still in its infancy in practical applications, thus it's bound to have some problems to be studied and solved, which would restrict the application of micro arc oxidation. 
Therefore micro-arc oxidation technology should be continuously studied and improved to enable it to meet the actual needs of the practical application, and only by doing this micro-arc oxidation technology can have a broader application prospect in the field of aviation engines.

\section{References}

[1] GONG Weixing. Research on Preparation and Corrosion Resistance of Micro-arc Oxidation Coatings of Magnesium Alloy and Magnesium Matrix Composite[D]. Harbin:Harbin Engineering University, 2012.

[2] BIN Yuanhong, LIU ying, LI Peifen. The Effects of Magnesium Alloy MAO Coatings with Different Frequency and Duty Ratio[J]. Science Technology and Engineering 2011, 11(31): 76407643.

[3] WU Junyi, LI Zhongsheng, WU Hulin, et al. Investigation on Electroless Ni-P-SiC Composite Plating on Micro-arc Oxidation Film of Aluminium Alloy[J]. Surface Technology, 2013, 42(4): $101-106$.

[4] WANG Zhigang, CHEN Weidong, YAN Shufang, et al.Microstructure and Hydrogen Permeation Resistance of Zirconium Hydride Coated by Micro Arc Oxidation Process in Na5P3O10 Solution[J]. Rare Metal Materials and Engineering, 2015(11): 2877—2881.

[5] PANG Liuyang. The Application of Aluminum Alloy Microarc Oxidation in Military Parts[J]. New Technology \& New Process, 2009(2): 29—31. 